Classification

Physics Abstracts

$61.30 \mathrm{E}-64.70 \mathrm{E}$

\title{
A new thermotropic smectic phase made of ribbons (*)
}

\author{
F. Hardouin, Nguyen Huu Tinh, M. F. Achard and A. M. Levelut (**) \\ Centre de Recherche Paul-Pascal, Domaine Universitaire, Université de Bordeaux-I, 33405 Talence, \\ France
}

$\left.{ }^{(* *}\right)$ Laboratoire de Physique des Solides (***), Bât. 510, Université Paris-Sud, 91405 Orsay, France

(Reçu le 24 décembre 1981, révisé le 3 mars 1982, accepté le 15 mars 1982)

\begin{abstract}
Résumé. - L'étude des textures et de la structure des phases mésomorphes d'un nouveau composé polaire thermotrope, le nitrobenzoyloxybenzoate d'octylphényle nous a permis de mettre en évidence une nouvelle phase fluide constituée de rubans formant un réseau bidimensionnel oblique. Nous proposons un modèle simplifié basé sur un calcul de facteurs de structure.
\end{abstract}

\begin{abstract}
From microscopical observations and structural studies on the mesophases of a new thermotropic polar compound - the nitrobenzoyloxybenzoate of octylphenyl - we have shown the existence of a novel fluid mesophase built with ribbons forming a two-dimensional oblique lattice. We propose a crude model based upon structure factor calculations.
\end{abstract}

1. Introduction. - It is known that mesogen compounds with a terminal cyano-end group can exhibit a rich polymorphism such as reentrant sequences [1] or $S_{A}-S_{A}$ transitions [2]. The second sequence has been found only for the cyanobenzoyloxybenzoates of alkyl phenyl $\mathrm{DB}_{n}$ and for their mixtures with other mesogens $[3,4,5]$. A transition between two $S_{A}$ phases corresponds to a change in the layer thickness of the smectic layer, the liquid-like order in a layer remains of the same kind. The substitution of the cyano group by a nitro group induces for the heptyl derivative $\left(\mathrm{DB}_{7} \mathrm{NO}_{2}\right)[6]$ a new kind of phase in which a two-dimensional centred rectangular lattice is superimposed over a short range liquid-like order between adjacent molecules. We report here our investigations upon the polymorphism of the octyl nitro derivative $\left(\mathrm{DB}_{8} \mathrm{NO}_{2}\right)$ which is shown to exhibit a new two-dimensional phase. The study of this compound has been made by microscopical observation, thermal analysis, magnetic susceptibility measurements and X-ray diffraction.

2. Preliminary study. - The " $\mathrm{DB}_{8} \mathrm{NO}_{2}$ " compound

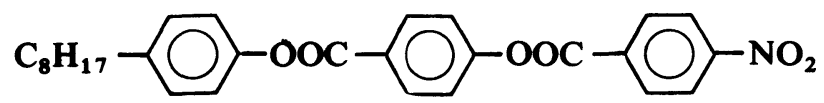

(*) La version française de cet article a été proposée pour publication aux Comptes Rendus de l'Académie des Sciences.

$\left({ }^{* * *}\right)$ Associé au C.N.R.S. 
exhibits the following mesophases :

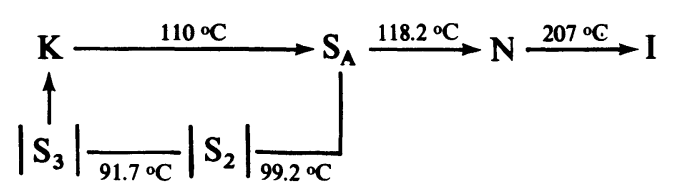

By decreasing temperature from a homeotropic domain of the $\mathrm{S}_{\mathrm{A}}$ phase we observe at $T_{\mathrm{S}_{\mathrm{A}} \mathrm{S}_{2}}$ the occurrence of a transient texture (Fig. $1 b$ on left). This texture rapidly disappears giving a stable texture formed by polydomains (Fig. $1 c$ on left). Just before the crystallization, at $T_{\mathrm{S}_{2} \mathrm{~S}_{3}}$ a homeotropic texture appears again. In the non homeotropic domains (Figs. 1a,1b,1c on right) one can see a mosaic texture in the $S_{2}$ temperature range (Figs. $1 b, 1 c$ on right). This sequence is corroborated by DSC measurements, in particular at $T_{\mathbf{S}_{\mathrm{A}} \mathrm{S}_{2}}$ a narrow heat peak corresponding to a transition entropy $\Delta S_{\mathrm{S}_{\mathrm{A}} \mathrm{S}_{2}}=260$ cal. mole ${ }^{-1} \mathrm{~K}^{-1}$ is detected.

Furthermore by Faraday's method [7] we have performed diamagnetic susceptibility measurements in a direction parallel to the magnetic field $\left(\chi_{\|}\right)$. At the $S_{A} \rightarrow S_{2}$ a dramatic increase of $\left|\chi_{\|}\right|$is revealed. In good agreement with the microscopic observations this result seems to indicate that the $S_{2}$ phase is not uniaxial.

3. X-ray diffraction studies. - The sample was contained in a Lindemann glass tube, a $0.3 \mathrm{~T}$ magnetic field orienting the director in the nematic phase, the X-ray beam coming from a doublebent pyrolitic graphite and the diffracted beams being collected on a flat film.

In the nematic phase we observed the usual large angle diffuse ring at $4.5 \AA$ and two diffuse spots aligned along the direction of the magnetic field, the two corresponding scattering vectors are $q_{1 z}=\frac{2 \pi}{51.6} \AA^{-1}$ and $q_{2 z}=\frac{2 \pi}{29.5} \AA^{-1}$. Cooling down in the $\mathrm{S}_{\mathrm{A}}$ phase the first diffuse spot becomes a Bragg spot while the second remains diffuse. From the position of the Bragg spot we can assert that we have a partially bilayer $\mathrm{S}_{\mathrm{A}}$ phase with a layer thickness $d \simeq 44.5,45 \AA \gg L(L$ molecular length obtained from stereomodel : $31 \AA$ ) $[4,5]$.

The diffuse spot takes the shape of a thin disc in the reciprocal space with a component along the director $q_{1 z}=\frac{2 \pi}{28.7} \AA^{-1}$ at $116^{\circ} \mathrm{C}$ and $q_{1 z}=\frac{2 \pi}{28} \AA^{-1}$ at $105^{\circ} \mathrm{C}$. This diffuse scattering comes from a short range order of periodic columns constituted with strings of molecules, thus the longitudinal period in the column is approximately equal to the molecular length.

At $T<99^{\circ} \mathrm{C}$ in the $S_{2}$ phase the small angle part of the X-ray pattern shows an important modification while the large angle diffuse ring remains unchanged (Fig. 2).

Let us call $\mathrm{O} z$ and $\mathrm{O} x$ two rectangular axes with $\mathrm{O} z$ parallel to the magnetic field. Increasing $q_{z}$ we see sharp spots lying respectively at :

$$
\mp q_{x}, q_{1 z} ; 0, q_{2 z} ; q_{x}, q_{3 z} ; 0,2 q_{2 z} ; q_{x}, q_{4 z} \text {. }
$$

Depending on the sample, the spots $\mp q_{x}, q_{z}$ have not the same intensity and sometimes only one set of spots is visible. Thus the $\mathrm{O} z$ axis is not a symmetry axis of the reciprocal lattice, we have an oblique $2 d$ lattice and a few single crystals are seen simultaneously. This observation confirms the biaxial behaviour previously observed. We can index the spots as $\overline{1} 1 ; 01 ; \overline{1} 2 ; 02 ; \overline{1}$. The 10 and 12 spots are not visible, the 11 spot is merely seen on the diffuse scattering background surrounding the intense 01 peak, the 13 spot can be seen only on highly exposed films. From the values of the scattering vectors we can deduce the lattice constants :

$$
a=106 \AA, \quad c=58 \AA, \quad \beta=120^{\circ}
$$


a)

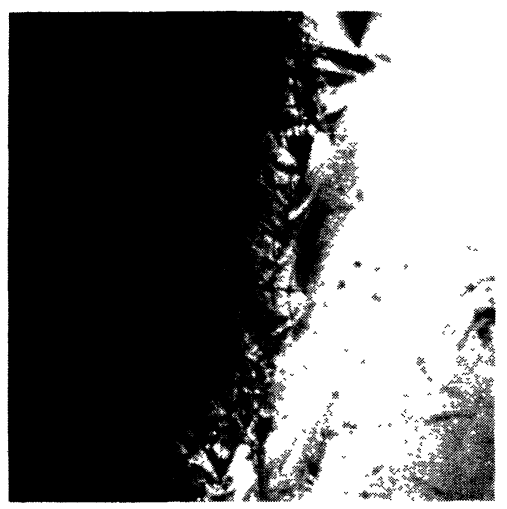

b)
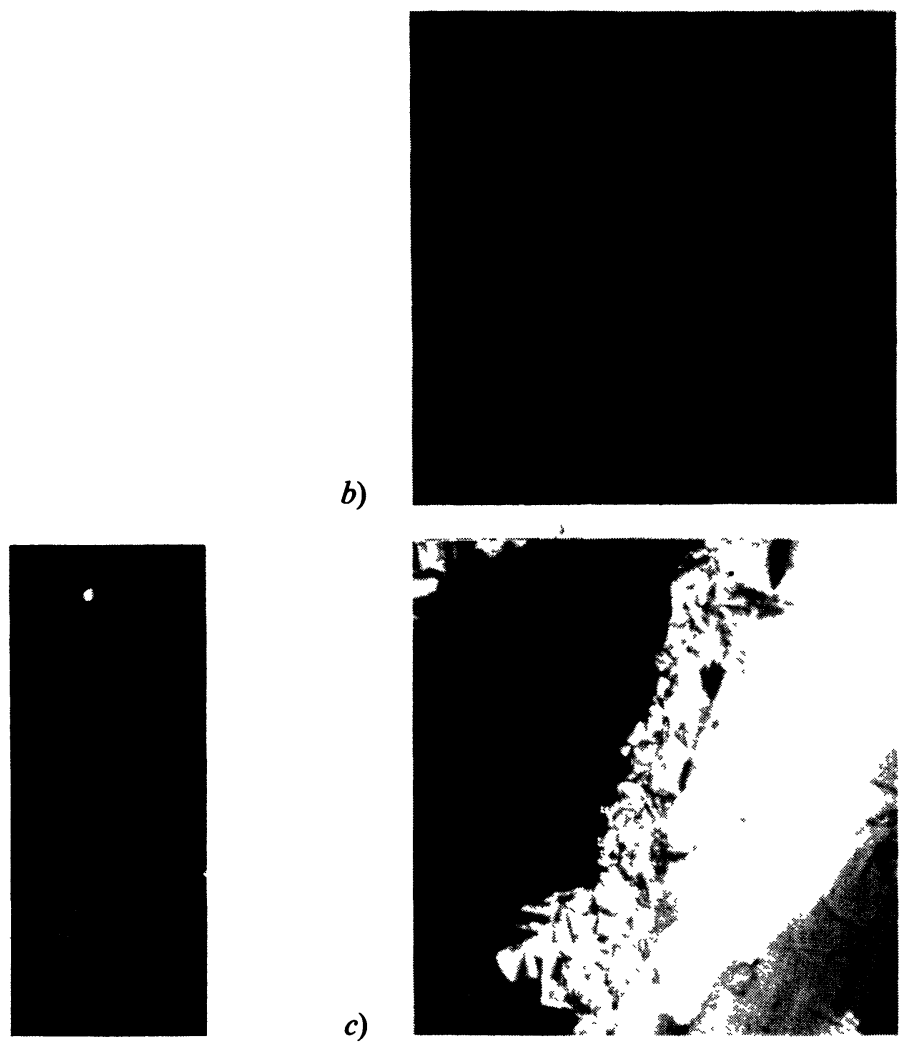

Fig. 1. - a) $\mathrm{DB}_{8} \mathrm{NO}_{2}$, smectic A, homeotropic domain on left, $T=99.3{ }^{\circ} \mathrm{C}(\times 200)$. b) $\mathrm{DB}_{8} \mathrm{NO}_{2}, \mathrm{~S}_{2}$ phase : transient texture on left, mosaic texture on right; $\left.T=99.2^{\circ} \mathrm{C}(\times 200) . c\right) \mathrm{DB}_{8} \mathrm{NO}_{2}, \mathrm{~S}_{2}$ phase : the transient texture disappears giving a stable texture (on left), mosaic texture on right; $T=99^{\circ} \mathrm{C}(\times 200)$.

the $a$ axis is perpendicular to the magnetic field while the $c$ axis makes a $30^{\circ}$ angle with the magnetic field. A similar structure has already been described by A. Tardieu [8] for some mesophases of synthetic lecithines. Such a phase was named the $\mathbf{P}_{\boldsymbol{\beta}^{\prime}}$ phase and it is formed by asymmetrically undulated layers. In the case of the $\mathbf{P}_{\beta^{\prime}}$ phase the paraffinic chains are rigid and form a hexagonal bidimensional array in the layers. On the other hand the centre of mass of the molecules in the $\mathrm{S}_{2}$ phase are randomly distributed within planes perpendicular to the director but we can keep the image of undulated layers. In order to have a better insight into the structure we have performed 


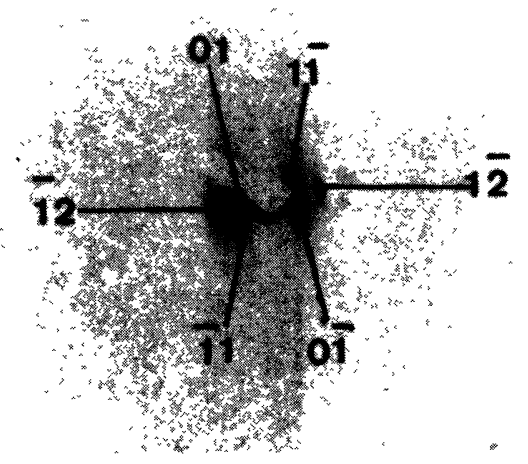

Fig. 2. - X-ray pattern of the $S_{2}$ phase (central part).

a structure factor calculation on a simplified model since we have just an idea of the order of magnitude of the diffracted intensities.

Taking into account earlier studies on $\mathrm{DB}_{n}$ and $\mathrm{DB}_{n} \mathrm{NO}_{2}$ series [3-6], we assume that the molecule form pairs which remain parallel to the magnetic field since there is no change in the large angle ring. If the centres of the pairs are on a plane $z=u x$ in reduced units the amplitude of the diffracted $\mathrm{X}$-ray is given by :

$$
F(h, l)=F_{\text {pair }}\left(q_{z}\right) \cdot \frac{\sin (\pi(h+u l))}{\pi(h+u l)}
$$

where $h$ and $l$ are the indices of the Bragg spots, $F_{\text {pair }}$ is the form factor of a pair of molecule, $q_{z}$ is the projection of the scattering vector along $c^{*}$. The length of the pair is deduced from the values of $c, \beta$ and $u$.

In table I we have reported the experimental and calculated values for the reticular distances and also the diffracted intensities, using $u=0.6$ which corresponds to the best fit. In figure 3 we give a schematic model for this structure. The layers are broken in ribbons of bimolecular pairs with periodic stacking faults. The amplitude of the displacement between two adjacent ribbons is 0.8 molecular length. In fact we can also describe this phase as a regular stacking of bimolecular tilted regions and regions in which the order of two adjacent ribbons are interlaced. The relative importance of the two regions depends on the $\beta$ value. We can compare this phase with the twodimensional phase of $\mathrm{DB}_{7} \mathrm{NO}_{2}\left(\mathrm{~S}_{\tilde{\mathrm{A}}}\right)$ [4]. In this latter case the lattice is centred rectangular (in the

Table I. - Reticular spacing and intensity of visible spots.

\begin{tabular}{ccc}
$h, k$ reflections & \multicolumn{2}{c}{ Reticular spacing $(\AA)$} \\
- & experimental & calculated \\
$\frac{10}{11}$ & - & - \\
01 & - & - \\
11 & 55.05 & 58.65 \\
$\overline{1} 2$ & 49.15 & 49.15 \\
02 & - & 35.57 \\
12 & 27.92 & 27.92 \\
$\overline{13}$ & 24.58 & 24.58 \\
& - & 20.93 \\
& 17.84 & 17.95
\end{tabular}

Intensity (arbitrary unit) calculated

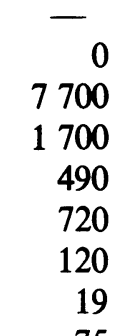




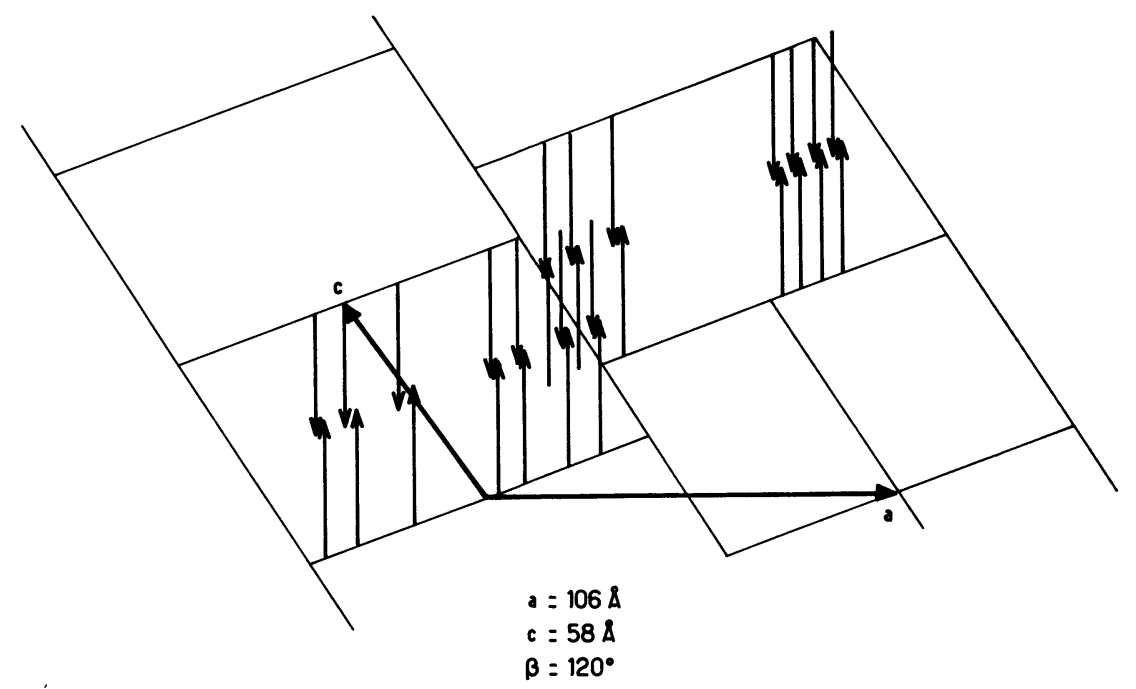

Fig. 3. - Schematic model for the structure of the $S_{2}$ phase.

limit of accuracy of our X-ray measurements). In accordance with the symmetry of the lattice we have proposed for $\mathrm{DB}_{7} \mathrm{NO}_{2}$ a ribbon structure in which the ribbon of bimolecular pairs has a rectangular section. The centred rectangular array of such a ribbon is equivalent to a bilayered structure broken periodically by defects walls. The two sides of the wall are translated by one half of the layer thickness, i.e. one molecular length. $T$ The $S_{\widetilde{A}}$ phase is similar to the antiphase observed in some alloys. Therefore the difference between the two-dimensional phases of $\mathrm{DB}_{7} \mathrm{NO}_{2}$ and $\mathrm{DB}_{8} \mathrm{NO}_{2}$ comes from the difference in the respective orientations of the walls, director and layers. In $\mathrm{DB}_{7} \mathrm{NO}_{2}$ the director is parallel to the walls and perpendicular to the layers while the direction of both the walls and the layers are oblique with respect to the director in $\mathrm{DB}_{8} \mathrm{NO}_{2}$. We have also to note that the thickness of each wall is important compared to the width of each ribbon since no high order of reflection has been observed in planes perpendicular to the director in $\mathrm{DB}_{7} \mathrm{NO}_{2}$ as well as in $\mathrm{DB}_{8} \mathrm{NO}_{2}$. That assumption is consistent with our model of the structure of the $\mathrm{S}_{2}$ phase of $\mathrm{DB}_{8} \mathrm{NO}_{2}$. By analogy with the antiphase we propose to name this serrated phase a $\mathrm{S}_{\mathfrak{c}}$ phase. This nomenclature underlines the fact that this two-dimensional fluid structure is a tilted one.

Unfortunately, before crystallization we cannot detect by X-ray experiments the metastable $\mathrm{S}_{3}$ phase.

Acknowledgments. - We are grateful to P. Barois, C. Coulon and Dr. J. Prost and Dr. G. Sigaud for valuable discussions.

\section{References}

[1] See for example Sigaud, G., Nguyen huU tinh, Hardouin, F., Gasparoux, H., Mol. Cryst. Liq. Cryst. 69 (1981) 81.

[2] Sigaud, G., Hardouin, F., Achard, M. F., Gasparoux, H., J. Physique Colloq. 40 (1979) C3-356.

[3] Hardouin, F., Levelut, A. M., Benattar, J. J., Sigaud, G., Solid State Commun. 33 (1980) 337.

[4] Hardouin, F., Levelut, A. M., Sigaud, G., J. Physique 42 (1981) 71.

[5] Levelut, A. M., Tarento, R. J., Hardouin, F., Achard, M. F., Phys. Rev. A 24 (1981) 2180.

[6] Hardouin, F., Sigaud, G., NguYen huU tinh, Achard, M. J., J. Physique-Lett. 42 (1981) L-63.

[7] Achard, M. F., Hardouin, F., Sigaud, G., Gasparoux, H., J. Chem. Phys. 65 (1976) 4.

[8] TARDIEU, A., Thesis, Orsay (1972). 